\title{
Radiation History Negative
}

National Cancer Institute

\section{Source}

National Cancer Institute. Radiation History Negative. NCI Thesaurus. Code C160215.

There is no history of radiation exposure. 